\title{
Comprometimento e Entrincheiramento Organizacional: Possíveis Correlações
}

\section{Organizational Commitment And Entrenchment: Possible Correlations}

\author{
Jaqueline Cavalcante Milhome ${ }^{1}$, Diva Ester Okazaki Rowe ${ }^{1}$ \\ ${ }^{1}$ Universidade Federal da Bahia, UFBA, Brasil. \\ Correspondência: Jaqueline Cavalcante Milhome. Ondina, Salvador, CEP 40.170-115, Salvador, BA, Brasil. \\ Telefone: +55 (71) 3283-7072. E-mail: jaquemilhome@ gmail.com.
}

Recebido: 17 de agosto de 2018 Aceito: 17 de outubro de 2018 Publicado: 28 de dezembro de 2018

DOI: http://dx.doi.org/10.21714/1679-18272018v16n1.p69-77

\begin{abstract}
Resumo
Os estudos dos vínculos com a organização vêm amplamente buscando compreender o Comprometimento e o Entrincheiramento Organizacional enquanto construtos independentes, investigando possíveis correlações e influências entre eles. Esta pesquisa tem por objetivo central verificar o grau de correlação entre os referidos construtos, uma vez que dizem respeito à diferentes (não opostas) naturezas de ligação do trabalhador com a organização e coexistem no trabalhador. Para alcançar o referido objetivo utilizou-se correlação de Kendall, por ser adequada à amostras não probabilísticas, bem como ao tamanho da amostra desta pesquisa. Para que fossem analisados os construtos, utilizou-se a média ponderada para chegar a um valor representativo do nível de Comprometimento Organizacional e de cada uma das dimensões do Entrincheiramento Organizacional. O peso utilizado para o cálculo da média ponderada foi a carga fatorial. Os resultados provenientes desta análise confirmam parcialmente as hipóteses desta pesquisa, levantadas a partir do referencial teórico e apontaram correlação muito baixa entre os construtos, em ambas as análises. Quando considerada a amostra como um todo, os dados indicam que os custos com investimentos de adaptação contribuem positivamente para o Comprometimento Organizacional. Porém, quando essa análise considera os níveis de Comprometimento Organizacional, percebe-se que esta correlação somente evidencia-se significativamente em trabalhadores com baixo nível de Comprometimento Organizacional.
\end{abstract}

Palavras-chave: Comprometimento Organizacional, Entrincheiramento Organizacional, Vínculos com a organização.

\begin{abstract}
Studies about links towards organizations have sought broadly to understand Organizational Commitment and Organizational Entrenchment like independents constructs, exploring possible relationships and influences among themselves. This research has main objective to verify correlation standard between Organizational Commitment and Organizational Entrenchment, because they are related to different (not opposite) kinds of worker link toward organization, which coexist at workers. To arrive the research objective, it was used Kendall's correlation, because it is appropriated to no-probabilistic sample and the sample size of this research. To analyze the constructs, was used weighted average to find out a representative score to level of Organizational Commitment and each factor of Organizational Entrenchment. The factor value used to weighted average was a coefficient by exploratory factor analysis. The results of this research confirmed partially the ts hypothesis, elucidated by theories. Its indicates very low correlation between constructs, in both analysis. Considering all sample, the data indicate that adaptation pain costs contribute positively Organizational Commitment. However, in a level of Organizational Commitment section analysis, is perceived that the correlation is only significantly evidenced at low level of Organizational Commitment workers.
\end{abstract}

Keywords: Organizational Commitment, Organizational Entrenchment, Links toward Organizations.

Esta obra está licenciada sob uma Licença Creative Commons Attribution 3.0.

\section{Introdução}

As relações que os trabalhadores estabelecem com a organização vêm sendo estudadas há alguns anos, sob diversas perspectivas. Busca-se compreender como essas relações se estabelecem, o que contribui para que se estabeleçam 
e o que estas relações geram ou influenciam.

No que diz respeito ao Comprometimento Organizacional, as discussões provenientes dos diversos estudos levaram a dois modelos preponderantes, mas divergentes entre si, no que tange suas premissas. Um deles aponta o Comprometimento Organizacional como um vínculo de natureza afetiva, enquanto o outro, que ganhou mais destaque especialmente no decorrer das décadas de 1990 e 2000, difundia que este vínculo é também, afetivo, porém, somado ao elemento instrumental e normativo, compondo-se um vínculo tridimensional.

Ademais, discussões mais recentes, como Cunha et al. (2004), Rodrigues (2009) e Solinger; Olffen; Roe (2008), seguindo recomendações de Osigweh (1989), contestam o modelo tridimensional, defendendo que cada uma dessas três dimensões, diante dos seus antecedentes e consequentes tão diferentes, bem como do que definem e representam em si, trata-se, na verdade, de vínculos diferentes. Dois destes vínculos são analisados nesta pesquisa: Comprometimento Organizacional e Entrincheiramento Organizacional. Sobre estes, pergunta-se: em que medida os vínculos comprometimento organizacional e entrincheiramento organizacional se relacionam?

O Comprometimento Organizacional é compreendido como um vínculo de natureza afetiva que se caracteriza pelo orgulho de pertencimento, uma identificação com normas e procedimentos, afetividade pela organização. Ao passo que o Entrincheiramento Organizacional diz respeito a um vínculo estabelecido a partir das necessidades e possíveis perdas do trabalhador (BASTOS et al., 2013). Enquanto o primeiro está relacionado com comportamentos desejados, como a contribuição ativa para o alcance das metas e comportamento de cidadania organizacional, o segundo está vinculado a comportamentos não desejáveis, como absenteísmo, negligência baixo desempenho, maior intenção de saída e menor centralidade no trabalho (BANDEIRA; MARQUES; VEIGA, 2000; REGO; SOUTO, 2004; WASTI, 2005).

Estudos realizados com estes dois vínculos conjuntamente indicaram que eles não são independentes entre si, existindo concomitantemente no trabalhador, em diferentes níveis (BALSAN et al., 2015; MEIRELES, 2015; RODRIGUES, 2009). Dessa forma, é importante analisar se o crescimento de um acarreta uma variação do outro, em algum sentido, indicando a existência de alguma relação entre eles.

Considerando a referida coexistência dos vínculos no trabalhador, perceber em que medida o aumento de um vínculo está relacionado ao aumento ou diminuição do outro ajuda a compreender como o estímulo de um pode potencializar ou diminuir a existência do outro no indivíduo. Aos gestores, por sua vez, entender como ocorre essa relação permitirá uma atuação mais propensa a incentivar comportamentos esperados e diminuir comportamentos não desejáveis, contribuindo para melhores respostas na organização, tanto em termos de resultados como acerca da relação das pessoas na organização em si.

Meireles et al. (2015) e Rodrigues (2009), realizaram análises similares. No entanto, este estudo realizou esta investigação a partir de uma metodologia diferente dos demais, apropriada às características da sua amostra, e em uma perspectiva nova, em comparação aos demais. Uma nova análise em amostra tão diversificada e diferente das anteriormente estudadas, considerando metodologias diferentes, é capaz de corroborar e ampliar a percepção acerca de relações entre vínculos com os trabalhadores.

O objetivo central desta pesquisa consistiu em verificar o grau da relação entre o Comprometimento Organizacional e o Entrincheiramento Organizacional. Para tanto, foi desmembrado em dois objetivos específicos: a) Verificar a correlação entre o comprometimento e as diferentes dimensões do entrincheiramento na amostra como um todo; b) Verificar a correlação entre comprometimento e as diferentes dimensões do entrincheiramento organizacional considerando subgrupos amostrais, a partir dos diferentes níveis de comprometimento e entrincheiramento organizacional.

Para apresentar os resultados deste estudo, este artigo foi organizado em seções. Após a introdução, será apresentado o referencial teórico, indicando possíveis relações entre os vínculos estudados. Em seguida, serão descritos os procedimentos metodológicos, apresentados e discutidos os resultados e, por fim, elucidadas as considerações finais.

\section{Vínculos Do Trabalhador Com A Organização: Comprometimento E Entrincheiramento}

Vidal e Rodrigues (2016) apresentam o vínculo do trabalhador com a organização como processos de troca, material e simbólica, cuja, "de um lado está a organização com suas metas e razões de existência social e, do outro, o indivíduo com seus desejos e anseios". Está ligado a diversos elementos "(envolvimento, identificação, apego, satisfação, motivação, centralidade, entre vários outros)" (MOSCON; BASTOS; JANISSEK, 2012) e permeia as relações do trabalhador com a organização. Desde os primeiros estudos realizados sobre vínculos, entre as décadas de 1950 e 1960, pesquisas buscaram defini-los e compreender, especialmente, o que causa e o que é consequência destes (RODRIGUES, 2011). 
Com o avanço dos estudos, modelos foram propostos, estudados foram revisados. O Comprometimento Organizacional, um dos vínculos mais amplamente estudados, tem adotado como agenda de pesquisa, estudos que ajudem a compreender o que efetivamente corresponde ao Comprometimento Organizacional e o que diz respeito a outro tipo de vínculo, que precisa ser devidamente estudado a parte do Comprometimento Organizacional (RODRIGUES; BASTOS, 2015).

Estudos realizados por Rodrigues $(2009 ; 2011)$ apresentaram evidências empíricas de que as naturezas afetiva e instrumental dos vínculos organizacionais dizem respeito à construtos diferentes - Comprometimento e Entrincheiramento Organizacional, com diferentes antecedentes e consequentes.

O Comprometimento Organizacional tem sido compreendido como um vínculo afetivo com a organização e relaciona-se a comportamentos desejados, de contribuição ativa para o alcance das metas. Trata-se de um construto unidimensional e compreendê-lo perpassa por analisar variáveis ligadas ao orgulho de pertencer à organização, ao significado que a organização tem na vida do trabalhador, à preocupação do trabalhador com problemas e destino da organização e a de objetivos, valores e normas compartilhados. (BASTOS et al., 2013; BASTOS; AGUIAR, 2015; BECKER; ULLRICH; DICK, 2013; COOPER-HAKIM; VISWESVARAN, 2005).

Ao passo que o Entrincheiramento Organizacional é um vínculo instrumental, tridimensional, diz respeito ao trabalhador sentir-se preso à organização e está ligado a comportamentos não desejados, por parte dos trabalhadores. As três dimensões que explicam esse vínculo são denominadas: Ajustes Burocráticos Impessoais (ABI), Ajustamento à Posição Social (APS) e

Limitação de Alternativas(LA) (MEIRELES, 2015; RODRIGUES, 2011). A Figura1 apresenta as definições dos construtos e de suas dimensões.

Figura 1: Definições de Comprometimento, Entrincheiramento Organizacional e dimensões do Entrincheiramento Organizacional

\section{Comprometimento Organizacional}

Vínculo afetivo ligado ao compartilhamento de valores e metas, que refere-se à ideia de que o indivíduo preocupa-se com resultados positivos e bem-estar da organização, culminando em implicações positivas para a organização e o trabalhador. (PINIIO; BASTOS, 2014; BASTOS ET AL, 2013).

\section{Entrincheiramento Organizacional}

Trabalhador sentir-se preso à organização - trincheira - por necessidade ou busca por maior segurança e estabilidade, atrelado à diminuição de chances de perda. Essas perdas podendo ser financeiras, em relação ao cargo, ao trabalho, às redes de contatos, entre outras que restringem a percepção de alternativas de emprego que as supram. (MEIRELES, 2015; RODRIGUES, 2009; RODRIGUES; BASTOS, 2015).

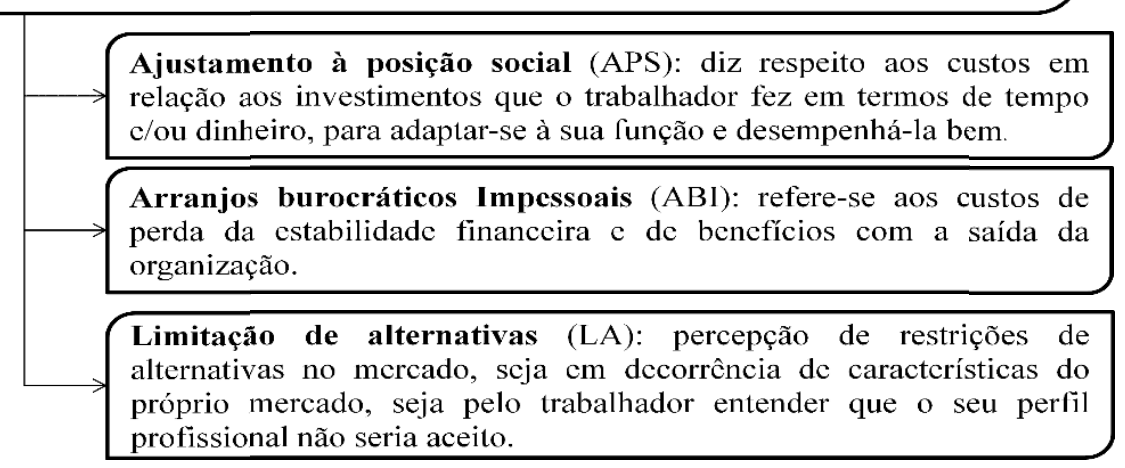

Fonte: BASTOS et al, 2013; MEIRELES, 2015; PINHO; BASTOS, 2014; RODRIGUES, 2009; RODRIGUES;

\section{BASTOS, 2015.}

Além das diferenças conceituais, estudos realizados por Balsan et al. (2015), Meireles (2015) e Rodrigues (2009; 2011) mostram diferenças empíricas entre o Comprometimento e o Entrincheiramento Organizacional. Trata-se de construtos com baixas correlações entre eles, bem como alta correlação entre a dimensão instrumental do Comprometimento Organizacional e o Entrincheiramento Organizacional.

Rodrigues (2009) trouxe uma discussão de inconsistências do modelo de Comprometimento Organizacional proposto por Meyer e Allen (1991), construindo o conceito de Entrincheiramento Organizacional a partir do que 
se define como Entrincheiramento na Carreira. Partindo dessa discussão inicial, foi construída uma escala para mensurar o Entrincheiramento Organizacional, e verificada a correlação entre as dimensões deste novo vínculo e a dimensão de continuação do Comprometimento Organizacional, segundo o modelo de Meyer e Allen. Foi encontrada correlação de 0,95 , indicando que se trata de um mesmo construto, por sua vez, diferente do vínculo afetivo. Em continuidade à esse estudo, Rodrigues (2011) confirmou a sobreposição indicada no estudo anterior.

Balsan et al. (2015) realizou pesquisa buscando relações existentes entre as bases do Comprometimento e Entrincheiramento Organizacional. Encontraram moderada correlação das bases instrumental e normativa do Comprometimento Organizacional, bem como baixa correlação entre a base instrumental e a base afetiva. Além disso, não se verificou correlação entre o Comprometimento Afetivo e o Entrincheiramento Organizacional, mostrando tratar-se, na realidade, de três vínculos.

Estudos que investigaram possíveis relações entre ambos os construtos encontraram correlação baixa entre os vínculos instrumental e afetivo do trabalhador com a organização. Balsan et al. (2015) encontrou correlações baixas, positivas e significativas, no que diz respeito às dimensões afetiva e instrumental do Comprometimento Organizacional, conforme defendido nos modelos propostos por Meyer e Allen. Rodrigues (2009) encontrou correlações um pouco maiores e igualmente significativas entre estas dimensões. Os resultados encontrados pelas diversas pesquisas que investigaram as diferenças entre as naturezas dos vínculos contribuíram para elucidar a primeira hipótese do presente estudo:

H1 - Existe uma relação baixa, positiva e significativa entre o Comprometimento Organizacional e as dimensões do Entrincheiramento Organizacional.

Em seus estudos, Rodrigues (2011) evidenciou, ainda, que esses vínculos não se estabelecem no indivíduo de forma isolada e sem correlações. Uma vez se tratando de vínculos diferentes, mas não opostos, a autora apresenta a existência conjunta destes no trabalhador, em maior ou menor grau. No entanto, pesquisas anteriores já buscavam compreender a combinação dos diferentes níveis da natureza afetiva e instrumental do vínculo do trabalhador com a organização.

Altos níveis de vínculo afetivo combinados com menores níveis de vínculo instrumental resultaram em comportamentos desejáveis. Maiores níveis de um vínculo instrumental prediziam baixo desempenho, maior intenção de saída e menor centralidade no trabalho (BANDEIRA; MARQUES; VEIGA, 2000; REGO; SOUTO, 2004; WASTI, 2005).

Considerando os diferentes resultados encontrados por pesquisas que analisaram os diferentes níveis de ambas as naturezas de vínculos coexistindo no trabalhador, considerando-as ou não diferentes construtos, elucidou-se a segunda hipótese desta pesquisa:

H2 - Em diferentes niveis de Comprometimento, as correlações entre o Comprometimento Organizacional e as dimensões do Entrincheiramento Organizacional apresentam diferenças.

Analisando outros elementos relacionados à satisfação com o trabalho, práticas de gestão e práticas de desenvolvimento profissional, Rodrigues (2011) percebeu que existem evidências de que estes vínculos atuam conjuntamente. No entanto, ressalta a possibilidade de outras variáveis não consideradas no estudo moderarem ou intervirem no aumento ou diminuição destes vínculos no trabalhador.

Menor satisfação com o trabalho e avaliações mais críticas das práticas de desenvolvimento profissional estão associadas aos indivíduos menos comprometidos, com baixo ou médio entrincheiramento. Indivíduos pouco satisfeitos com o trabalho e com o desenvolvimento profissional proporcionado pela organização tendem, portanto, a buscar alternativas de emprego. Por outro lado, as práticas de gestão voltadas para o desenvolvimento profissional e para maior satisfação com o trabalho geram comprometimento, mas não impactam isoladamente no nível de entrincheiramento do trabalhador (RODRIGUES, 2011).

Nas próximas sessões, serão analisadas possíveis correlações entre o Comprometimento Organizacional e as dimensões do Entrincheiramento Organizacional, a fim de testar se a coexistência dos construtos no trabalhador pode representar, também, relação entre eles.

\section{Procedimentos Metodológicos}

A presente pesquisa é quantitativa e descritiva. Segundo Gil (2008), a pesquisa descritiva objetiva descrever características de dada população ou fenômeno ou estabelecer relações entre variáveis. Enquadra-se nesta tipologia de pesquisa uma vez que o objetivo central desta pesquisa é relacionar dimensões de dois construtos.

Utilizou-se, como estratégia metodológica, o survey. Foram aplicados questionários on-line distribuídos por email e por rede social, com a utilização do Survey Monkey. O questionário foi composto de itens fechados, com 
escalas tipo Likert. Trata-se de uma escala intervalar que varia entre a discordância (menor valor) e a concordância (maior valor), existindo ou não um ponto neutro. Hair Jr. et al. (2009) defende o aumento da fidedignidade da escala, a partir da retirada do ponto neutro. Este procedimento foi utilizado nas escalas utilizadas para a coleta de dados, mantidas integralmente conforme validadas.

Além dos dados sociodemográficos, o questionário foi composto por duas escalas tipo Likert de 6 pontos: (a) A "Medida de Comprometimento Organizacional (MCO)", validada para trabalhadores brasileiros por Bastos e Aguiar (2015), composta por 12 itens; e (b) A "Medida de Entrincheiramento organizacional (MEO)", validada para trabalhadores brasileiros por Rodrigues e Bastos (2015), composta por 18 itens em três dimensões.

O questionário foi distribuído por e-mail para trabalhadores com vínculo formal de trabalho com organizações públicas ou privadas, independente da área de atuação. Além disso, o link foi disponibilizado em rede social, a fim de maior alcance da pesquisa. Diante da dificuldade para a obtenção de retorno, foi necessária aplicação de questionário impresso e pessoalmente. As respostas desses questionários foram inseridas diretamente no Survey Monkey, a fim de que as respostas fossem todas exportadas diretamente do programa. A coleta foi realizada entre os meses de setembro e dezembro de 2015.

O critério para definir a amostra foi a partir da disponibilidade das pessoas. Dessa forma, trata-se de uma amostragem não aleatória por conveniência, não sendo necessária a análise da normalidade, uma vez que esta é uma análise não representativa da população (HAIR et al., 2009).

Foram respondidos 284 questionários. As respostas foram exportadas para o Excel, a fim de serem iniciadas as análises. As análises de missing values e outliers foram realizada no software R. A análise de missing values considerou passível de exclusão todos os casos em que não houve preenchimento de alguma das escalas integralmente. Excluídos estes casos, realizou-se análise de outliers, a partir de boxplot. Não havendo nenhum caso a ser excluído, partiu-se para a análise dos coeficientes de variância. Trata-se de uma medida de dispersão utilizada para estimar a precisão de pesquisas, a partir de um percentual de variabilidade das respostas. (MAHALLEM et al., 2008).

Foram eliminadas respostas cujo coeficiente de variação fosse inferior a 0,05 e analisadas caso a caso, respostas com coeficiente de variação entre 0,05 e 0,09 . Totalizou 8 respostas, com base neste critério, considerando as respostas do construto Comprometimento Organizacional e 7 respostas quando analisadas as respostas do Entrincheiramento Organizacional.

Após essas análises prévias, a amostra final foi composta por 191 respostas, foi verificado a curtose e a assimetria. Todas as variáveis tiveram valor de assimetria acima do recomendado. No entanto, este já era um resultado esperado, devido o padrão de respostas para estes construtos. Portanto, optou-se por manter todas as variáveis e as respostas a serem analisadas.

A amostra, não probabilística, foi prioritariamente composta por trabalhadores de organizações privadas $(70 \%)$, solteiros (56\%), com idade variando entre 25 e 30 anos ( $42 \%$ ), sexo feminino (56\%), com formação superior (39\%) ou especialização (36\%).

A partir desta amostra, seguiu-se com o tratamento dos dados, utilizando o software R versão 3.3.2. Para testar as hipóteses desta pesquisa, correlacionaram-se as dimensões dos construtos estudados, utilizando correlação de Kendall. Optou-se por esta correlação porque esta não exige normalidade dos dados e seu resultado não é influenciado pelo aumento do tamanho da amostra.

No entanto, para ser possível correlacionar as dimensões, foi necessário chegar a valores que representassem cada dimensão dos construtos estudados, em lugar de valores correspondentes à cada resposta de cada variável. Este valor foi alcançado a partir da média ponderada das variáveis de cada caso correspondentes às dimensões dos construtos.

O peso utilizado para a média ponderada tomou como base a carga fatorial de cada variável, verificada a partir de análise fatorial exploratória. Uma vez calculadas as médias ponderadas, foi possível chegar a um valor que representasse o conjunto de respostas de cada caso, para cada uma das dimensões investigadas. Os resultados provenientes do tratamento dos dados serão apresentados na próxima seção.

\section{Apresentação e Discussão Dos Resultados}

Buscando atender ao objetivo central deste estudo, serão apresentados os resultados das correlações de Kendall. Este método, conforme descrito anteriormente, foi escolhido por ser adequado às características da amostra e ao objetivo.

A análise e compreensão das possíveis correlações entre Comprometimento e Entrincheiramento Organizacional iniciaram pela análise fatorial exploratória. Como se busca correlacionar os construtos, não as variáveis, é 
necessário que exista um valor que represente o nível de Comprometimento e o nível de cada uma das dimensões do Entrincheiramento, considerando o quanto suas variáveis representam o construto como um todo. Para tanto, obteve-se a média ponderada de cada resposta, sendo os pesos de ponderação da média definidos pelas cargas fatoriais de cada variável nas respectivas dimensões que representavam.

A partir da média ponderada, foram verificados dois modelos. Primeiro foi testada a correlação entre as dimensões do construto na amostra como um todo. O segundo testou essa correlação em diferentes grupos, considerando o nível de Comprometimento Organizacional (alto, médio e baixo comprometimento). A tabela 1 mostra os graus de correlação testados no primeiro modelo.

Tabela 1: Correlação entre Comprometimento Organizacional e Entrincheiramento Organizacional

\begin{tabular}{l|l|l}
\hline \multirow{2}{*}{} & \multicolumn{1}{l}{ CO } \\
\cline { 2 - 3 } & Tau de Kendall & p-value \\
\hline LA & $-0,0906$ & 0,0684 \\
\hline ABI & -0.0023 & 0.9630 \\
\hline APS - Ajustamento a Posição Social & 0.178 & 0.00031 \\
\hline \multicolumn{2}{r}{ Fonte: dados da pesquisa }
\end{tabular}

p-value $<0,05, \mathrm{CO}$ - Comprometimento Organizacional; LA - Limitação de alternativas; ABI - Ajustes Burocráticos Impessoais; APS - Ajustamento à Posição Social

A Tabela1 apresenta baixa correlação entre os construtos investigados. Somente a dimensão APS (Ajustamento à Posição Social) do Entrincheiramento Organizacional tem correlação significativa com o Comprometimento Organizacional. Esta diz respeito aos investimentos do trabalhador em termos de tempo e/ou dinheiro, para adaptar-se à sua função e desempenhá-la bem. Logo, os dados indicam que, em alguma medida, os custos com investimentos de adaptação contribuem positivamente para o Comprometimento Organizacional, bem como trabalhadores comprometidos podem ter.

A correlação entre o vínculo afetivo e os esforços de adaptação ao trabalho e à organização, sem que este vínculo esteja correlacionado com as demais variáveis do Entrincheiramento Organizacional, foi encontrada também por Balsan et al. (2015). Ao correlacionar as dimensões do Entrincheiramento Organizacional com as dimensões afetiva, instrumental e normativa do Comprometimento Organizacional, os autores encontraram correlação baixa e positiva entre a dimensão afetiva do Comprometimento Organizacional e a dimensão API (Ajustamento à Posição Inicial), cuja, segundo Bastos et al. (2008), diz respeito aos investimentos necessários à adaptação e bom desempenho do trabalhador na organização. As demais dimensões do Entrincheiramento Organizacional não apresentavam correlações significativas com a dimensão afetiva do Comprometimento Organizacional, bem como sua dimensão instrumental apresentava correlação entre moderada e alta com todas as dimensões do Entrincheiramento Organizacional.

De forma complementar, analisou-se, separadamente, as correlações entre Comprometimento Organizacional e as dimensões do Entrincheiramento Organizacional em grupos com diferentes níveis de Comprometimento Organizacional (baixo, médio e alto nível de Comprometimento Organizacional). Os resultados dessas correlações, apresentados na Tabela 2, foram similares à primeira correlação, em um dos grupos. No entanto, somente é indicada correlação entre APS e CO no grupo com baixo comprometimento.

Tabela 2: Correlação entre Comprometimento e Entrincheiramento Organizacional em diferentes níveis de Comprometimento.

\begin{tabular}{l|l|l|l|l|l|l}
\hline & \multicolumn{3}{l}{ Baixo CO } & \multicolumn{2}{l}{ Médio CO } & \multicolumn{2}{l}{ Alto CO } \\
\hline & Tau de Kendall & p-value & Tau de Kendall & p-value & Tau de Kendall & p-value \\
& & & & & & \\
\hline LA & 0,0111 & 0,94528 & -0.0498 & 0.48202 & -0.122 & 0.16536 \\
\hline ABI & 0,0344 & 0,79874 & -0.0901 & 0.19926 & -0.0661 & 0.44773 \\
\hline APS & 0,521 & 8,2596 e-05 & 0.0316 & 0.65451 & -0.0987 & 0.2571 \\
\hline
\end{tabular}

Fonte: Dados da pesquisa

p-value $<0,05$; CO - Comprometimento Organizacional; LA - Limitação de alternativas; ABI - Ajustes Burocráticos Impessoais; APS - Ajustamento à Posição Social

Os resultados apresentados nos dois modelos confirmam parcialmente as hipóteses. A Hipótese H1 (Existe uma relação baixa, positiva e significativa entre o Comprometimento Organizacional e as dimensões do Entrincheiramento Organizacional) foi parcialmente confirmada uma vez que uma das três correlações (CO e 
APS) apresentou-se baixa, positiva e significativa.

Por sua vez, a Hipótese $\mathrm{H} 2$ (Em diferentes níveis de Comprometimento, as correlações entre o Comprometimento Organizacional e as dimensões do Entrincheiramento Organizacional apresentam pouca diferença) foi também parcialmente confirmada. No entanto, somente observou-se correlação positiva e significativa, também se tratando de APS, quando se analisou o grupo de trabalhadores com nível de comprometimento baixo. Neste caso, a correlação foi mais forte do que a apresentada no modelo1.

Rodrigues (2011) justifica essa correlação forte com aspectos que tangem os relacionamentos interpessoais "ou mesmo ao processo de identificação que pode estar associado à adaptação do indivíduo à sua organização". Nesta amostra é possível inferir que trabalhadores com menor nível de comprometimento percebem, proporcionalmente, menor custo ligado a relacionamentos interpessoais ou a esforços de adaptação à organização ou ao trabalho. Porém, esta correlação não foi percebida, com significância estatística, nos demais grupos.

O trabalhador comprometido vincula-se afetivamente com a organização, compartilhando valores e metas e se preocupando com resultados positivos e bem estar da organização. Um baixo nível de comprometimento, estatisticamente, significa que este grupo de trabalhadores apresentou média de respostas inferior a 2,5, o que indica que houve discordância em relação a todos os itens ou à grande maioria deles.

Analisando os itens da escala, isso significa que este trabalhador tende a não reconhecer uma ligação afetiva nem ter orgulho de trabalhar na organização, não compartilhar os valores, objetivos e formas de pensar da organização, não concordar com as normas, ser indiferente aos problemas da organização.

Os dados desta pesquisa indicam que trabalhadores com este perfil consideram baixo o custo de perder relacionamentos interpessoais construídos no ambiente de trabalho, bem como os esforços e/ou custos de adaptação à organização ou ao trabalho possuem pouca relevância. Dessa forma, a sensação de estar preso à organização devido a esses custos é pequena.

Como não foram evidenciadas outras relações significativas nesta amostra, não é possível afirmar que existe relação entre o Comprometimento (considerando a amostra como um todo e seus diferentes níveis) e o Entrincheiramento Organizacional. Conforme apontado por Rodrigues (2011), é possível que existam outras variáveis que interfiram nesta relação.

\section{Considerações finais}

A forma, a intensidade e a natureza do vínculo que o trabalhador estabelece com a organização influenciam diretamente as atitudes e os comportamentos do trabalhador. Portanto, esta pesquisa apresenta a correlação entre dois vínculos que vêm sendo amplamente estudados, devido sua importância no ambiente de trabalho.

O Comprometimento Organizacional, compreendido como um vínculo preponderantemente afetivo, está ligado a comportamentos adequados, ao passo que o Entrincheiramento Organizacional leva o trabalhador a comportamentos não desejados pela organização.

Esta pesquisa buscou as possíveis relações entre os vínculos investigados, analisando se existem correlações entre estes e em qual intensidade. A partir dessa análise, será possível contribuir para melhor compreensão sobre estes, especialmente o Entrincheiramento Organizacional, estudado enquanto construto há pouco tempo.

A pesquisa contou com uma importante limitação: o tamanho da amostra. Apesar de a amostra permitir que as análises fossem feitas com representatividade estatística, uma maior amostra poderia evidenciar outras correlações possivelmente significativas, especialmente nos subgrupos de diferentes níveis de Comprometimento Organizacional. Ainda assim, conclusões interessantes foram possíveis a partir desses dados.

Os resultados indicaram a correlação de somente uma dimensão do Entrincheiramento Organizacional com o Comprometimento Organizacional. Esta dimensão diz respeito aos custos ligados aos relacionamentos interpessoais e aos esforços despendidos para adaptação no trabalho - APS. Apresentou-se uma correlação considerada moderada, quando da análise da amostra como um todo, aumentando o grau de correlação e melhorando a significância estatística quando as variáveis foram correlacionadas somente nos casos com baixo nível de comprometimento.

Por um lado, é possível dizer que os resultados desta pesquisa, em grande medida, corroboram com os estudos que vêm sendo realizados, fortalecendo a ideia de que os vínculos afetivo e instrumental dizem respeito a construtos diferentes, em contraposição ao modelo tridimensional do Comprometimento. Porém, conforme demonstram algumas dessas pesquisas, ainda que em pequena medida, existe alguma correlação entre os construtos.

Assim, mostra-se interessante compreender melhor as possíveis influências no que diz respeito aos diversos níveis de Comprometimento Organizacional e Entrincheiramento Organizacional entre eles e, principalmente, o que a 
combinação desses diferentes níveis representa para a organização. Relacionar os construtos com elementos ligados ao indivíduo, não à organização, como valores pessoais ou personalidade. E que essas pesquisas pudessem perceber, inclusive, essa relação dentro dos diferentes níveis de comprometimento, entrincheiramento e dos grupos identificados por Rodrigues (2011).

\section{Referências}

BALSAN, L. A. G.; BASTOS, A. V. B.; FOSSÁ, M. I. T.; LIMA, M. P.; LOPES, L. F. D.; COSTA, V. M. F. Comprometimento e Entrincheiramento Organizacional: Explorando as Relações entre os Construtos. Revista de Administração da UFSM, v. 8, n. 2, p. 235-248, 2015.

BANDEIRA, M. L.; MARQUES, A. L.; VEIGA, R. T. As dimensões múltiplas do comprometimento organizacional: um estudo na ECT/MG. Revista de Administração Contemporânea, v. 4, n. 2, p. 133-157, 2000.

BAstos, A. V. B.; ROdRIGUES, A. C. A.; MOSCON, D. C. B.; SILVA, E. E. C.; PINHO, A. P. M. Comprometimento no trabalho: fundamentos para a gestão de pessoas. In: BORGES, L. O.; MOURÃO, L. (Org.). O trabalho e as organizações: atuações a partir da psicologia. Porto Alegre: Artmed, 2013

BASTOS, A. V. B. AGUIAR, C. V. N. Comprometimento organizacional. In: Kátia Puente- Palacios; Adriano de Lemos Alves Peixoto. (Org.). Ferramentas de diagnóstico para organizações e trabalho: Um olhar a partir da psicologia. 1ed. Porto Alegre: Artmed, 2015.

BECKER, T. E.; ULLRICH, J.; DICK, R. V. Within-person variation in affective commitment to teams: Where it comes from and why it matters. Human Resource Management Review, v. 23, p. 131-147, 2013.

COOPER-HAKIM, A.; VISWESVARAN, C. The construct of work commitment: testing an integrative framework. Psychological Bulletin, v. 131, n. 2, p. 241-259, 2005.

CUNHA, M. P.; REGO, A.; CUNHA, R. C.; CABRAL-CARDOSO, C. A ligação pessoa organização. In: CUNHA, M. P.; REGO, A.; CUNHA, R. C. M. P. (Orgs.). Manual de comportamento organizacional, Lisboa: Editora RH, p. 147-192, 2004.

GIL, A. C. Como elaborar projetos de pesquisa. 4. ed. São Paulo: Atlas, 2008

HAIR, J. F., JR.; ANDERSON, R. E.; TATHAM, R. L.; BLACK, W. C. Análise multivariada de dados. Porto Alegre: Bookman, 2009.

MEIRELES, A. Q. Fico porque preciso: entrincheiramento organizacional e satisfação no trabalho para empregados de uma empresa de energia brasileira. Dissertação (Mestrado em administração) Departamento de Administração, Pontifícia Universidade Católica do Rio de Janeiro, Rio de Janeiro, 169 p., 2015.

MEYER, J. P.; ALLEN, N. J. A three-component conceptualization of organizational commitment. Human Resource Management Review, v. 1, p. 61-89, 1991.

MOHALLEM, D. F.; TAVARES, M.; SILVA, P. L.; GUIMARÃES, E. C.; FREITAS, R. F. Avaliação do coeficiente de variação como medida da precisão em experimentos com frangos de corte. Arquivo Brasileiro de Medicina Veterinária e Zootecnia, v.60, n.2, p.449-453, 2008.

MOSCON, D. C. B.; BASTOS, A. V. B.; JANISSEK-DE-SOUZA, J. A. É possível integrar, em um mesmo conceito, os vínculos afetivo e instrumental? O olhar de gestores sobre o comprometimento com a organização. Organizações \& Sociedade (O\&S), v.19, n.61, 2012.

OSIGWEH, C. A. B. Concept fallibility in organizational science. The Academy of Management Review, v. 14, n. 4, p. 579-594, 1989.

PINHO, A. P. M., BASTOS, A. V. B. Vínculos do trabalhador com a organização: Comprometimento, entrincheiramento e consentimento. São Paulo: Hucitec, 2014.

REGO, A.; SOUTO, S. A percepção de justiça como antecedente do comprometimento organizacional: um estudo luso-brasileiro. Revista de Administração Contemporânea, v. 8, n. 1, p. 151-177, 2004.

RODRIGUES, A. C. A. Do comprometimento de continuação ao entrincheiramento organizacional: o percurso de validação da escala e análise da sobreposição entre os construtos. Dissertação (Mestrado em Psicologia), Instituto de Psicologia, Universidade Federal da Bahia, 2009.

RODRIGUES, A. C. A. Trabalhador entrincheirado ou comprometido? Delimitação dos vínculos do indivíduo 
com a organização. Tese (Doutorado em Psicologia), Instituto de Psicologia, Universidade Federal da Bahia, 2011.

RODRIGUES, A. C. A.; BASTOS, A. V. B. Entrincheiramento Organizacional. In: PUENTE-PALACIOS, K.; PEIXOTO, A. L. A. (Org.). Ferramentas de diagnóstico para organizações e trabalho: Um olhar a partir da psicologia. 1ed.Porto Alegre: Artmed, 2015.

SOLINGER, O. N.; OLFFEN, W.; ROE, R. A. Beyond the three-component model of organizational commitment. Journal of Applied Psychology, v. 93, n.1, p. 70-83, 2008

VIDAL, D. N.; RODRIGUES, A. P. G. Vínculos Organizacionais: estudo de caso no $13^{\circ}$ batalhão de Bombeiros Militares. Revista Gestão e Planejamento, Salvador, v. 17, n. 1, p. 4- 18, jan./abr. 2016.

WASTI, S. A. Commitment profiles: Combinations of organizational commitment forms and job outcomes. Journal of Vocational Behavior, n. 67, p. 290-308, 2005. 